\title{
Advanced cyclostationary-based analysis for condition monitoring of complex systems
}

\author{
Konstantinos Gryllias \\ Department of Mechanical Engineering \\ KU Leuven \\ DMMS Group, Flanders Make \\ Leuven, Belgium \\ konstantinos.gryllias@kuleuven.be
}

\author{
Alexandre Mauricio \\ Department of Mechanical Engineering \\ $K U$ Leuven \\ DMMS Group, Flanders Make \\ Leuven, Belgium \\ alex.ricardomauricio@kuleuven.be
}

\author{
Junyu Qi \\ Department of Mechanical Engineering \\ KU Leuven \\ DMMS Group, Flanders Make \\ Leuven, Belgium \\ junyu.qi@kuleuven.be
}

\begin{abstract}
Wind energy experiences a significant growth during the last decades but the industry is still challenged by premature turbine component failures, which are quite expensive due to the increase of turbines size. The core of wind turbine drivetrains is a planetary gearbox and its rolling element bearings are often responsible for machinery breakdowns. The failure signs of an early bearing damage are usually weak compared to the gear excitation and are hardly detected. As a result there is a special need for advanced signal processing tools which can detect accurately bearing faults. Cyclic Spectral Coherence (CSC) appears to be a strong diagnostic tool but its interpretation is complicated for a non-expert. In this paper a novel CSC based methodology is proposed in order to extract an Improved Envelope Spectrum exploiting a specific domain of the CSC map optimally selected by a proposed criterion. The methodology is tested and validated on a wind turbine gearbox benchmarking dataset provided by the National Renewable Energy Laboratory (NREL), USA.
\end{abstract}

Index Terms-Signal Processing, Cyclostationary Analysis, Cyclic Spectral Coherence, Condition Monitoring, Fault detection

\section{INTRODUCTION}

Wind energy is an emerging market for power generation but needs to become more cost effective in order to increase its competitiveness as an extra advantage to the clean energy production. This can be significantly succeeded by the increase of the availability and the reliability using condition monitoring methodologies. The National Renewable Energy Laboratory (NREL), USA performed a round robin condition monitoring measurement campaign [1], [2] on a wind turbine in order to test the capabilities of existing vibration based detection methods. In the last decade a plethora of methodologies have been proposed focusing towards the accurate and early fault detection and diagnosis with a minimum of false alarms and missed detections. One of the most commonly used methods is Envelope Analysis [3], where the vibration signal is demodulated using often the Hilbert Transform. The signal is usually filtered around an excited resonance of the machinery

The authors would like to gratefully acknowledge Flanders Make and VLAIO Flanders Innovation and Entrepreneurship Agency within the VIBMON - Cost-effective vibroacousic monitoring project for their support. K. Gryllias would like to gratefully acknowledge the Research Fund KU Leuven. The authors would like to thank the National Renewable Energy Laboratory (NREL), USA. in order to increase the Signal-to-Noise Ratio (SNR). In order to select accurately the filter bandwidth, a parameter is usually optimised such as the Spectral Kurtosis, which leads to the Kurtogram [4], and the Peak Energy Criterion [5]. Moreover Antoni [6], [7] proposed the use of CSC as a method for the spectral analysis of cyclostationary vibration signals emitted by rolling element bearings. CSC can be accompanied by different prewhitening [8] and Random/Periodic separation algorithms [9]. The integration of CSC over all the frequency band leads to the Enhanced Envelope Spectrum (EES). The aim of this paper is the introduction of a new automated methodology for the extraction of a filtered version of EES, named the Improved Envelope Spectrum (IES), using a maximization criterion, focusing towards the enhancement of the available diagnostic information. The structure of the paper is as follows. The theory of the Cyclic Spectral Analysis is briefly presented in Section II. The NREL wind turbine test rig is presented in Section III. The methodology is applied in Section IV and the results are analysed. The paper closes with some conclusion in Section V.

\section{CYCLIC SPECTRAL ANALYSIS}

Cyclostationary signals, although they are not necessarily periodic, are produced by a hidden periodic mechanism. The cyclostationarity category includes stationary signals (assuming an infinite period), non-stationary signals which exhibit periodicity after passing through a non-linear transform and periodic signals as a special case, describing practically a series of mechanical signals generated by rotating and reciprocating machines. A signal is characterised as cyclostationary of order $n$ when its $n$th order statistics are periodic. A firstorder cyclostationary signal (CS1) presents a steady first-order statistics, i.e the mean value in the ensemble average sense is unaltered [6], [7], i.e its first-order moment or expected value $m_{x}(t)$ is periodic with period $\mathrm{T}$.

$$
m_{x}(t)=\mathbb{E}\{x(t)\}=m_{x}(t+T)
$$

In rotating machines, $\mathrm{CS} 1$ vibrations are periodic waveforms (e.g. as generated by imbalances, misalignments, anisotropic rotors, flexible coupling etc.) with possibly additive stationary random noise. Similarly, a second-order cyclostationary signal 
(CS2) has an autocorrelation function which is a periodic function of time:

$$
R_{x x}(t, \tau)=\mathbb{E}\{x(t-\tau / 2) x(t+\tau / 2)\}=R_{x x}(t+T, \tau)
$$

The symmetric-lag product convention has been followed in this paper. When available for all values of $\mathrm{t}$ and $\tau$, the autocorrelation function presented in (2) contains all the information about a second-order cyclostationary signal and the representation in the frequency domain usually provides more insight into the structure of the signal. In rotating machines, CS2 vibrations are stochastic processes with periodic amplitude or/and frequency modulation (e.g. as generated by wear, friction forces, impacting forces, fluid motions, turbulence, combustion forces, etc). Signals that are CS1 and CS2 are referred to as wide-sense cyclostationary. Since the autocorrelation function is a function of two variables, a twodimensional Fourier transform is performed, giving rise to the so-called "spectral correlation":

$$
S_{x x}(\alpha, f)=\lim _{W \rightarrow \infty} \frac{1}{W} \int_{\mathbb{R}} \int_{-W / 2}^{W / 2} R_{x x}(t, \tau) e^{-j 2 \pi(f \tau+\alpha t)} d t d \tau
$$

This involves two frequency variables with very different physical meanings. Frequency $f$, as being the dual of timelag $\tau$, indicates the frequency of the carrier signal. Frequency $\alpha$, as being the dual of time $t$, indicates the frequency of the modulation and, accordingly, is usually named the "cyclic frequency" or the "modulation frequency". Hence, the spectral correlation may be interpreted as giving the strength of the elementary waves in signal $x$ carried and modulated at all possible combinations $(\alpha, f)$. The autocorrelation function of a second-order cyclostationary signal is transient in time-lag $\tau$ and periodic in time $t$ and as a result the corresponding spectral correlation is continuous in $f$ but discrete in $\alpha$, returning a very distinctive signature. Alternatively the spectral correlation can be calculated as:

$$
S_{x x}(\alpha, f)=\lim _{T \rightarrow \infty} \mathbb{E}\left\{X_{T}(f+\alpha / 2) X_{T}^{*}(f-\alpha / 2)\right\}
$$

where $X_{T}(f)$ stands for the Fourier transform of signal $x(t)$ over an interval of duration T. The 2D plan of Cyclic Spectral Correlation is a powerful tool containing all the available diagnostic information but often an expert is needed to interpret it. On the other hand, the integral of the Spectral Correlation over all frequencies $f$ is the Fourier transform of the expected value of the squared signal and as a result it corresponds to the Spectrum of the Squared Envelope of the signal:

$$
\begin{array}{r}
\int S_{x x}(\alpha, f) d f=\iint \mathbb{E}\left\{x(t+\tau / 2) x^{*}(t-\tau / 2)\right\} \\
\left(\int e^{-2 j \pi f t} d f\right) e^{-j 2 \pi \alpha t} d t d \tau= \\
\iint \mathbb{E}\left\{x(t+\tau / 2) x^{*}(t-\tau / 2)\right\} \delta(\tau) d \tau e^{-j 2 \pi \alpha t} d t= \\
\int \mathbb{E}\left\{x(t) x^{*}(t)\right\} e^{-j 2 \pi \alpha t} d t=\mathcal{F}\left\{\mathbb{E}\left\{|x(t)|^{2}\right\}\right\}
\end{array}
$$

Moreover the Cyclic Spectral Coherence can be estimated as a normalized version of the Cyclic Spectral Correlation with its magnitude normalized within 0 and 1 :

$$
\gamma_{x x}(\alpha, f)=\frac{S_{x x}(\alpha, f)}{\sqrt{S_{x x}(0, f) S_{x x}(0, f-\alpha)}}
$$

The Cyclic Spectral Coherence can be interpreted as the Spectral Correlation of a whitened signal, which tends to equalize regions with very different energy levels, magnifying weak cyclostationary signals. The Squared Envelope Spectrum, measured in a given frequency band $\left[f_{1}, f_{2}\right]$ can be also defined as:

$$
S_{x x}^{S E S}=\left|\int_{f_{1}}^{f_{2}} \gamma_{x x}(\alpha, f) d f\right|
$$

Furthermore the Enhanced Envelope Spectrum (EES) can be defined as:

$$
S_{x x}^{E E S}=\int_{f_{1}}^{f_{2}}\left|\gamma_{x x}(\alpha, f)\right| d f
$$

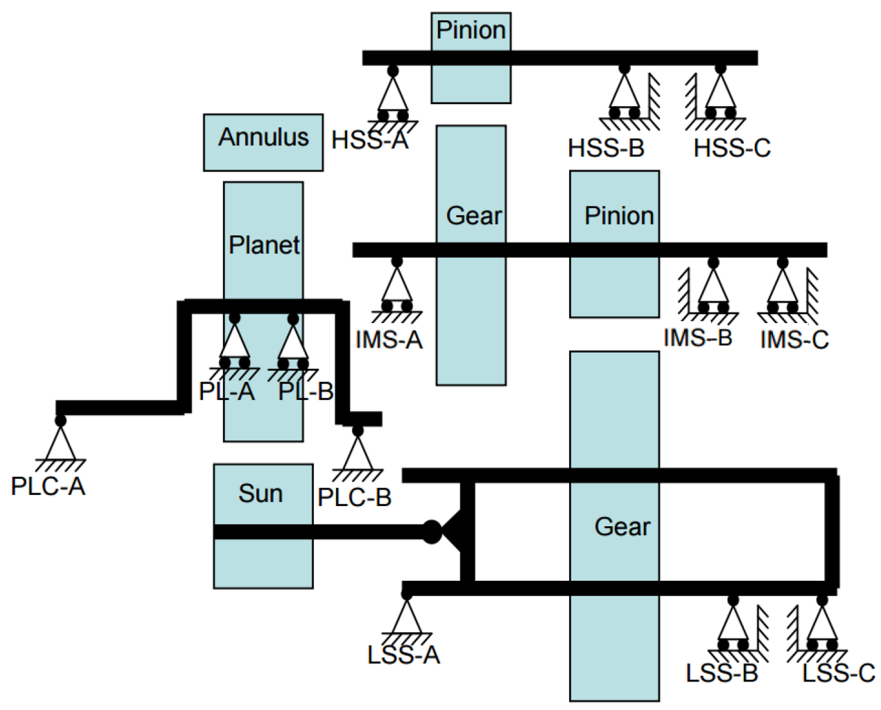

Fig. 1. Test gearbox nomenclature and bearing location.

The EES is expected to better enhance non-zero cyclic components compared to the classical SES as the latter integrates complex values and this can possibly converge towards zero in the case of fast rotating phases. The Envelope Spectrum can be estimated based on the integration simply over all the frequency band $\left[f_{1}=0, f_{2}=f_{s} / 2\right]$ or over a specially selected frequency band presenting a high $\operatorname{SNR}\left(f_{s}\right.$ is the sampling frequency).

In this work a novel criterion is proposed in order to optimally select the integration frequency band based on the (diagnostic) Feature Optimisation. The spectral frequency (y axis of the CSC map) is scanned sequentially with different bandwidths $\left[f_{1}, f_{2}\right]$. For each bandwidth the Cyclic Spectral Coherence is integrated in the specific limits band $\left[f_{1}, f_{2}\right]$ and 
the Improved Envelope Spectrum is estimated. The sum of the amplitude of the first three harmonics of each bearing characteristic frequency (BPFO, BPFI, BSF, FTF) is estimated and is used as a criterion for the quality of the envelope of the signal. The integration at specific frequency bands is equivalent to classical filtering and the maximisation of the sum of the amplitudes of the three harmonics of the defect is expected to provide an envelope spectrum with characteristic and well distinguished peaks. The value of the criterion is plotted as a function of the central frequency and finally the limits can be estimated. The steps of the methodology are briefly described in Fig. 2.



Fig. 2. Schematic of proposed methodology.

TABLE I

SHAFTS AND GEAR MESHING FREQUENCIES

\begin{tabular}{|l|r|r|}
\hline & Order $(/ H S S)$ & Freq. $(\mathrm{Hz})$ \\
\hline Rotor & 0.012 & 0.37 \\
\hline Planets & 0.043 & 1.30 \\
\hline Sun shaft & 0.070 & 2.10 \\
\hline Intermediate Shaft & 0.250 & 7.50 \\
\hline Generator Shaft (HSS) & 1.000 & 30.00 \\
\hline (GMF) Sun $\rightarrow$ IMS & 5.750 & 172.00 \\
\hline (GMF) IMS $\rightarrow$ HSS & 22.000 & 660.00 \\
\hline
\end{tabular}

GMF: Gear Meshing Frequency, HSS: High Speed Shaft,

IMS: Intermediate Speed Shaft.

\section{DESCRIPTION OF THE TEST RIG}

The aim of this paper is the proposal of a methodology for the accurate and early bearing fault detection and diagnosis. The methodology is tested and evaluated on a publicly available wind turbine gearbox vibration condition monitoring benchmarking dataset provided by the NREL after request, which investigated the root causes and loading conditions that result in the premature failure of wind turbine gearboxes through a consortium entitled the Gearbox Reliability Collaborative (GRC). In the frames of this initiative, NREL performed a benchmarking data campaign using the NREL Dynamometer Test Facility (DTF). The test turbine is a stallcontrolled three bladed upwind turbine with a rated power of $750 \mathrm{~kW}$. The turbine operates at $1800 \mathrm{rpm}$. The complete nacelle and drivetrain was installed in the NREL DTF and hard fixed to the floor without the hub, rotor, yaw bearing or yaw drives. The actual field controller was used to provide start-up and system safety responses. Two gearboxes, one "healthy" and one "damaged" of the same design have been tested under the GRC dynamometer and vibration data have been collected by accelerometers along with high-speed shaft RPM signals. The "healthy" gearbox was only tested at the dynamometer. The "damaged" gearbox first was run in the dynamometer and was later moved to a wind farm close to NREL for field testing. During the field test, the gearbox experienced two loss-of-oil events that damaged its internal bearings and gear elements. The gearbox was brought back to NREL and Condition Monitoring equipment was installed and then retested under controlled loading conditions that would not cause catastrophic failure of the gearbox. The two test gearboxes were originally taken from the field and redesigned with the specific configuration, rebuilt and instrumented. Both gearboxes have an overall ratio of 1:81.482. Each gearbox is composed of one low speed (LS) planetary stage (1:5.714) and two parallel stages (1:3.565 and 1:4.000). The nomenclature and the bearing location of the test gearboxes is presented in Fig. 1, while the shaft rotating speeds and the gear meshing frequencies can be found at Table I. Eight accelerometers (AN3 - AN10) mounted on the casing of the gearbox have been used to collect data with a sampling frequency equal to $40 \mathrm{kHz}$ per channel.

TABLE II

CHARACTERISTIC BEARING FAULT FREQUENCIES

\begin{tabular}{|l|r|r|c|}
\hline Bearing label & Fault type & Freq. $(\mathrm{Hz})$ & Sensor \\
\hline HSS Downwind & BPFI & 345.3 & AN7 \\
\hline HSS Downwind & FTF & 12.8 & AN7 \\
\hline IMS Downwind & BPFO & 105.3 & AN6 \\
\hline IMS Upwind & BPFI & 73.7 & AN6 \\
\hline PLC Upwind & BPFO & 8.8 & AN5 \\
\hline
\end{tabular} PLC: Planet Carrier, BPFI: Ball Pass Frequency Inner race,
BPFO: Ball Pass Frequency Outer race,
FTF: Fundamental Train Frequency.

\section{APPLICATION OF THE METHODOLOGY AND RESULTS}

The methodology presented in Section II has been tested and evaluated on signals from the benchmarking dataset presented in Section III. The "damaged" gearbox experienced two oilloss events in the field and was later disassembled in order to conduct a detailed failure analysis. A list of the bearing fault types and their corresponding characteristic frequencies occurred to the test rig is presented in Table III accompanied by the number of the closest sensor to the defect. The signals captured by the sensors close to each bearing are analysed in order to detect the faults. A number of researchers participating at the consortium have applied different methodologies [1] in order to detect the bearing faults but in the initial blind analysis they all failed in detecting the fault at the outer race of the Planetary Carrier upwind bearing, which was caused by fretting corrosion. The methodology has been applied on all the signals but here for space reasons, the application on the most difficult case is presented. 


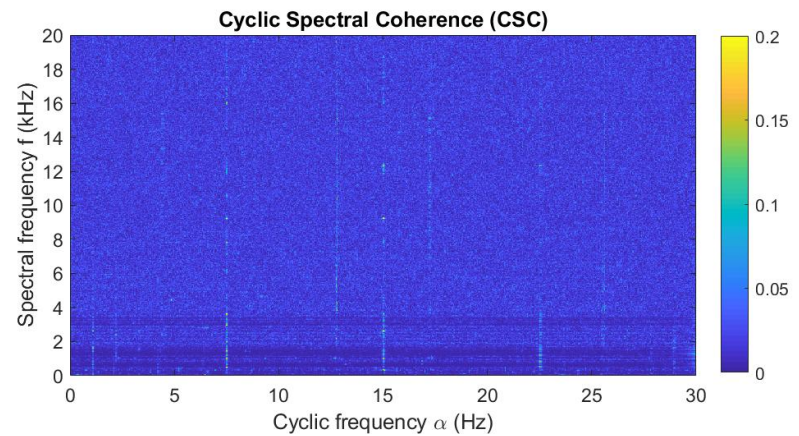

(a)

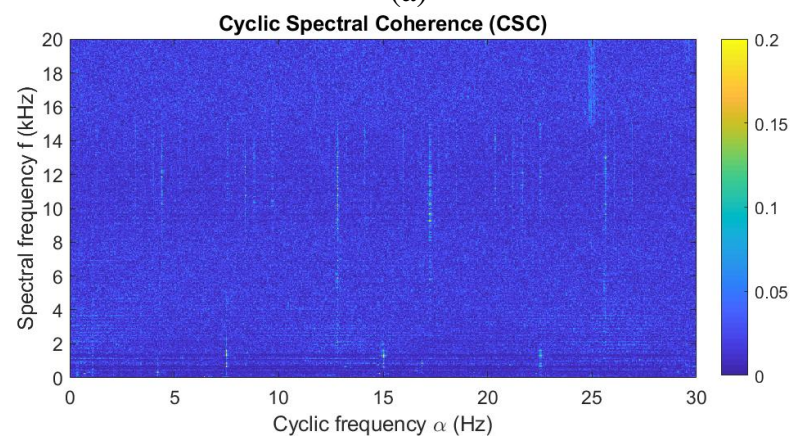

(b)

Fig. 3. CSC (a) Healthy case, (b) Damaged case.

First the time signals captured by the sensor AN5 in the healthy and the faulty conditions are processed and the CSC $2 \mathrm{D}$ maps are calculated. A zoom version in the bandwidth [0 $\mathrm{Hz}-30 \mathrm{~Hz}$ ] of the two CSC maps are presented at Fig. 3 (a) and (b). The harmonics of the intermediate shaft are present while a bearing characteristic frequency, demonstrating the presence of a bearing fault, cannot be clearly identified. The map contents all the diagnostic information but it is difficult to be revealed especially by a non expert. Next the Enhanced Envelope Spectrum is estimated by integrating over all the frequency bandwidth for both the healthy and the damaged case and are presented at Fig. 4 (a) and (b). The cyclic frequencies equal to the harmonics of the existing BPFO defect are marked with red dots. It is clear based on the figures that a detection cannot be easily and accurately achieved. Furthermore the Feature Optimisation criterion is applied and the sum of the amplitude of the first three harmonics of the defect is presented as a function of the central frequency of the signal in Fig. 5. The y-axis has no units, as the normalised amplitude is estimated based on the CSCoh map and therefore is a non-dimensional value. A filter with the central frequency $f_{c}$ and the bandwidth $B_{w}$ selected by the procedure are further applied on the spectral frequency (y axis) and an Improved Envelope Spectrum is estimated by the integration along the specific selected frequency band. The Improved Envelope Spectrum is presented at Fig. 6. Analysing the spectrum, the first three harmonics of the characteristic frequency BPFO are clearly identified, which could not be identified in the Enhanced Envelope Spectrum presented in



(a)



(b)

Fig. 4. EES (a) Healthy case, (b) Damaged case.

Fig. 4. This case is the most difficult one included in the specific dataset as the shaft rotating speed of the bearing is very low and as a result the signal which is emitted by the impacts of the rolling elements on the defect is very weak. The BPFO characteristic frequency of the Planetary Carrier upwind bearing takes a value close to the 4th harmonic of the sun shaft frequency which is equal to $8.4 \mathrm{~Hz}$. On the other hand based on the speed of the bearing as well as on the fact that the sun shaft frequency appears as a clear peak at the damaged EESFO next to the peak of the BPFO (thanks to the high resolution of the method), it can be concluded that the defect is accurately detected. The proposed methodology has been applied on all the bearing signals provided by NREL and all the defects have been successfully identified (but are not presented in this paper for space reasons).

Ten (10) signals have been captured and processed for each of the sensors mentioned in Table III. The methodology proposed in this paper has been applied on all bearing sgnals 
provided by NREL and has been also compared with the state of the art technique of Envelope Analysis. In this case, the signals are firstly analysed using the well known Kurtogram (Fast Kurtogram: FK) and based on the maximization of Spectral Kurtosis a filter band is selected. Then the Squared Envelope Spectrum (SES) is estimated. Often in order to enhance the effectiveness of the method a pre whitening step is applied, such as the Zero Cepstrum (ZK) method. The results in terms of fault detection are presented in Table ??. The proposed methodology achieves an average detection in 96 $\%$ of the cases detected accurately in the faults in the first 4 cases and failing in only $2 / 10$ signals in the more difficult case. On the other hand the Kurtogram seems to select each time a wrong filter band failing to detect the fault. Based on ?? it can be concluded that EESFO achieves very good results compared to other methods.

TABLE III METHODS COMPARISON

\begin{tabular}{|l|r|r|c|c|}
\hline Bearing label & Fault type & FK+SES & ZC+FK+SES & EESFO \\
\hline HSS Downwind & BPFI & $100 \%$ & $50 \%$ & $100 \%$ \\
\hline HSS Downwind & FTF & $0 \%$ & $0 \%$ & $100 \%$ \\
\hline IMS Downwind & BPFO & $0 \%$ & $0 \%$ & $100 \%$ \\
\hline IMS Upwind & BPFI & $40 \%$ & $40 \%$ & $100 \%$ \\
\hline PLC Upwind & BPFO & $0 \%$ & $0 \%$ & $80 \%$ \\
\hline Average & & $28 \%$ & $18 \%$ & $96 \%$ \\
\hline
\end{tabular}

PLC: Planet Carrier, BPFI: Ball Pass Frequency Inner race, BPFO: Ball Pass Frequency Outer race,

FTF: Fundamental Train Frequency.

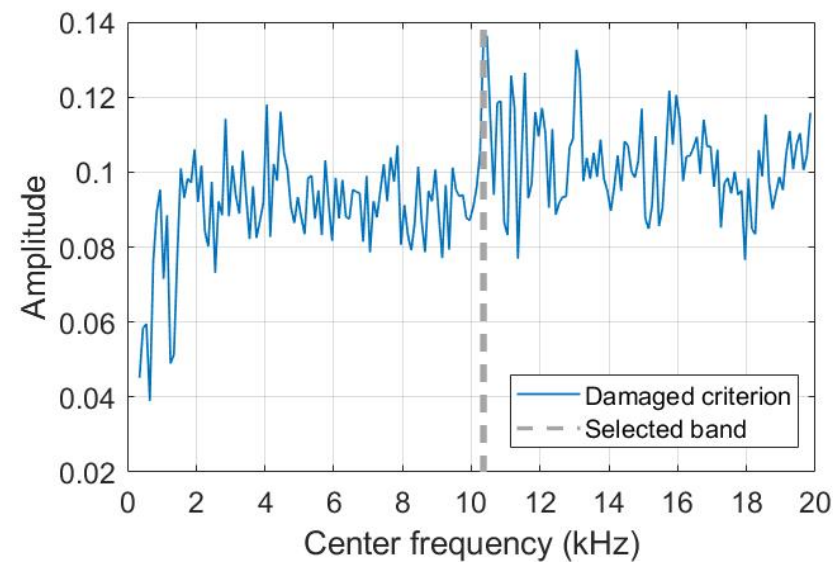

Fig. 5. Feature Optimisation Criterion.

\section{CONCLUSIONS}

A novel automated methodology for the extraction of the Improved Envelope Spectrum has been proposed based on the automated selection of an integration band, exploiting the maximization of a specific criterion which in this case is the sum of the amplitude of the first three harmonics of the characteristic bearing frequencies. The methodology is based on the Cyclic Spectral Coherence and has been tested and evaluated on a wind turbine gearbox benchmarking dataset



Fig. 6. Improved Envelope Spectrum based on the optimum band selection.

providing excellent results even in the worst case, which has been analysed in this paper. As future work, the authors are currently working towards the extension of the methodology in order to cover the case of time varying operating conditions of complex mechanical and mechatronic systems, such as the wind turbines and the gas turbines.

\section{REFERENCES}

[1] Sh. Sheng, "Wind Turbine Gearbox Condition Monitoring Round Robin Study Vibration Analysis," NREL/TP-5000-54530, 2012.

[2] Sh. Sheng, "Wind Turbine Gearbox Vibration Condition Monitoring Benchmarking Datasets," NREL/TP-5000-54530, 2012.

[3] R.B. Randall and J. Antoni, "Rolling element bearing diagnostics - A tutorial," Mechanical Systems and Signal Processing, vol. 25, pp. 485$520,2011$.

[4] J. Antoni and R.B. Randall, "The spectral kurtosis: application to the vibratory surveillance and diagnostics of rotating machines," Mechanical Systems and Signal Processing, vol. 20, pp. 308-331, 2006.

[5] K. Gryllias and I. Antoniadis, "A peak energy criterion (P.E.) for the selection of resonance bands in complex shifted morlet wavelet (CSMW) based demodulation of defective rolling element bearings vibration response," International Journal of Wavelets, Multiresolution and Information Processing, vol. 7, 2009.

[6] J. Antoni, "Cyclic spectral analysis in practice," Mechanical Systems and Signal Processing, vol. 21, pp. 597-630, 2007.

[7] J. Antoni, G. Xin and N. Hamzaoui, "Fast computation of the spectral correlation," Mechanical Systems and Signal Processing, vol. 92, pp. 248-277, 2017.

[8] R. Borghesani, P. Pennacchi, R. Randall, N. Sawalhi and R. Ricci, "Application of cepstrum pre-whitening for the diagnosis of bearing faults under variable speed conditions," Mechanical Systems and Signal Processing, vol. 36, pp. 370-384, 2013.

[9] R.B. Randall and N. Sawalhi, "Use of cepstrum to remove selected discrete frequency components from a time signal," Spring New York, New York, NY, pp. 451-461, 2011. 